\title{
Improving detection of buried land mines through sensor fusion
}

\author{
Brian A. Baertlein and Ajith Gunatilaka \\ The Ohio State University ElectroScience Laboratory \\ 1320 Kinnear Road, Columbus, OH 43212
}

\begin{abstract}
A sensor-fused system has been developed for detection of buried land mines. The system uses a ground-penetrating radar, an infrared camera, and an electromagnetic induction sensor. In the current implementation each sensor is used independently, and fusion is performed during post-processing. We briefly describe the sensors and a data collection involving buried mine surrogates. Algorithms for preprocessing and feature extraction are reviewed. To deal with non-coincident sampling we have developed a new feature-level fusion algorithm, which does not require detection and subsequent association of putative targets. Results are presented for fusion of simulated data and data measured in an outdoor surrogate minefield.
\end{abstract}

Keywords: Sensor fusion, land mines, ground-penetrating radar, infrared imaging, electromagnetic induction, metal detectors

\section{INTRODUCTION}

The potential benefits of sensor fusion for demining have prompted several groups to investigate this subject. Among the attributes claimed for sensor fusion are improved detection rates, decreased false alarm rates, and greater robustness in the face of instrument failure and diverse environmental conditions.

In this paper we describe the development of a sensor-fused system for detection of buried mines. Our system currently employs three sensors, which are operated independently on separate platforms. Fusion is performed during post-processing. A quantity of training data has been acquired using these sensors at our facility, where we have buried a number of mine surrogates.

Different sensor technologies tend to produce data which vary in their sampling of the area (e.g., point sensors versus imaging sensors), leading to non-coincident measurement locations. Differences in sampling locations are exacerbated when the sensors reside on different platforms. One method of dealing with non-coincident sampling is association, whereby putative detections from individual sensors are combined. In sensing buried mines, however, detection by individual sensors can be challenging and false alarm rates are typically high, making correct association difficult.

We have developed a novel technique for fusion which avoids the need for preliminary detection by individual sensors and is therefore better suited to irregularly sampled data. The algorithm is described herein, and results are presented for simulated sensor outputs and measured data.

This work is organized in five major sections. In Section 2 we describe the sensors used in the system. Data collection activities are reviewed in Section 3. Preprocessing algorithms required to prepare the data for fusion are given in Section 4. The fusion algorithm is described in Section 5. In Section 6 we present results of the fusion algorithm for a suite of three sensors.

\section{SENSORS}

To facilitate tests of our fusion concepts under a variety of conditions, a suite of sensors was acquired. Sensors used in the present system are a ground penetrating radar (GPR), a commercial infrared (IR) camera, and a Schiebel electromagnetic induction (EMI) sensor. Brief descriptions of these devices are presented below.

BAB (correspondence), voice (614) 292-0076, fax (614)-292-7297, E-mail: baertlein.1@osu.edu

An abbreviated version of this paper will appear in Detection and Remediation Technologies for Mines and Mine-Like Objects III, A. C. Dubey, J. F. Harvey, J. Broach (eds), SPIE Proceedings 3392, AeroSense98, Orlando, FL, April 1998. 


\subsection{Ground Penetrating Radar}

The GPR used in this effort was developed at The Ohio State University (OSU) ElectroScience Laboratory (ESL). It employs a single offset-fed, down-looking focussed parabolic reflector mounted on a wheeled platform. The antenna beam, which has a radius of 5 to 28 inches at the ground depending on frequency, is scanned in an arc over a distance of 38 inches. Complex (in-phase and quadrature) data are collected at 48 points along this arc. The transmitted waveform is frequency-stepped $\mathrm{CW}$ over the range 1-6 GHz in $100 \mathrm{MHz}$ steps. The antenna is positioned about four feet off the ground, which avoids direct antenna-ground coupling.

\subsection{Electromagnetic Inductions Sensor}

We use the Schiebel AN-19/2 metal detector, a pulsed induction sensor now deployed by the US military. This sensor consists of a search head with concentric circular transmit (inner) and receive (outer) coils. Approximate diameters of the coils are $18.5 \mathrm{~cm}$ and $28.5 \mathrm{~cm}$ respectively. The presence of a metal object is indicated by an audible tone of $1.3 \mathrm{kHz}$ in the user's earphone. As the search head approaches the metal object the amplitude of the tone and the harmonic content increase. Performance data quoted by the manufacturer are: detection of mines with very small $(0.15 \mathrm{~g})$ metal content at $10 \mathrm{~cm}$, and detection of a typical anti-tank mine at $50 \mathrm{~cm}$. The unit is supplied with a 0.15 g test piece, a steel pin of diameter $1.5 \mathrm{~mm}$ by $10 \mathrm{~mm}$ long (comparable to a Chinese Type $72 \mathrm{mine}$ ), which can be used to adjust its sensitivity. In addition to the standard audio tone, we have configured the instrument to provide a time-domain waveform output directly from the sensor head. This waveform includes the decay curve of the target, which (in principle) can be related to the shape and metal composition of the target.

\subsection{Infrared Camera}

The IR camera is an IRRIS 160ST produced by Cincinnati Electronics. This sensor has a InSb focal plane array of 160 by 120 12-bit pixels. It operates in the regime $2.2-4.6 \mu \mathrm{m}$, and it has a noise equivalent temperature difference $(\mathrm{NE} \Delta \mathrm{T})$ of $0.025 \mathrm{~K}$. The instantaneous field of view (IFOV) with the current optics is 1 mrad. The camera can be interfaced directly to a personal computer, with the computer performing camera control and image acquisition for long-term studies or for sampling rapid transient events at rates up to 160 frames per second.

\section{DATA COLLECTIONS}

Sensor data are required for training and testing a sensor fusion system. Using the sensors described above, data sets have been collected and are now being analyzed.

\subsection{Facility}

To better understand data artifacts and to explore "what-if" sensor questions, it is convenient to have ready access to a test mine field. For this reason a number of mine surrogates were buried in a level grassy field near the ESL facility. The buried surrogates are arranged in a 4-by-10 target grid as shown in Figure 1. Descriptions of the objects corresponding to the abbreviations in Figure 1 are given in Table 1. The objects include aluminum soft-drink cans at the corners, which are fiducial markers for the GPR and EMI sensors. White, reflective surface fiducials are also used when IR data is being collected.

In addition to the fiducials, the grid includes one mine surrogate of high metal content, four surrogates of low metal content, ten non-metal surrogates, a few man-made clutter features (crushed soda cans and "pop-tops"), and natural inhomogeneities (stones and voids).

\subsection{Data Acquisition}

Data were acquired over the mine grid for each of the three sensors. Sampling for each sensor is different, as is the format of the output data. Figure 2 illustrates the relation of the grid and the acquired samples.

The GPR platform was moved through the mine grid from East to West along each row of the grid. Data sets (arcs of 48 samples) were acquired every three inches starting from a point some distance to the east of the grid and ending about 15 inches to the west of the grid. Calibration data from a sphere placed above a microwave absorber background were also acquired for compensation of the system impulse response, but such compensation has typically not been required. Because of problems in precisely positioning a wheeled platform on uneven terrain, there is some error in the location of these points. In addition, a number of scans were lost when a data transfer failed without warning. 
Figure 1. The layout of the mine test grid. The key marked near each object is interpreted as the Abbreviation/Depth in inches.

Table 1. Description of the objects buried in the mine test grid

\begin{tabular}{|c|c|c|c|}
\hline Abbreviation & Object & $\begin{array}{c}\text { Diameter } \\
\text { (inches) }\end{array}$ & $\begin{array}{c}\text { Height } \\
\text { (inches) }\end{array}$ \\
\hline CC & Soda can & & \\
\hline CX & Crushed soda can & & \\
\hline PT & Pop top & & \\
\hline M35 & Aluminum disk & 3.5 & $5 / 8$ \\
\hline P15 & Plexiglass disk & 1.5 & 2 \\
\hline R4 & Rock & $\sim 4$ & $\sim 2$ \\
\hline V4 & Void(styrofoam) & $\sim 4$ & $\sim 2$ \\
\hline H4 & Refilled hole & $\sim 6$ & $\sim 2$ \\
\hline N35 & Nylon disk & 3.5 & $5 / 8$ \\
\hline N3D & Nylon disk with & 3 & 1 \\
\hline T3P & Teflon disk with & 3 & 1 \\
\hline T7 & .196 dia. 078 thick copper disk & & 1 \\
\hline N7 & Teflon disk & 7 & 1 \\
\hline
\end{tabular}




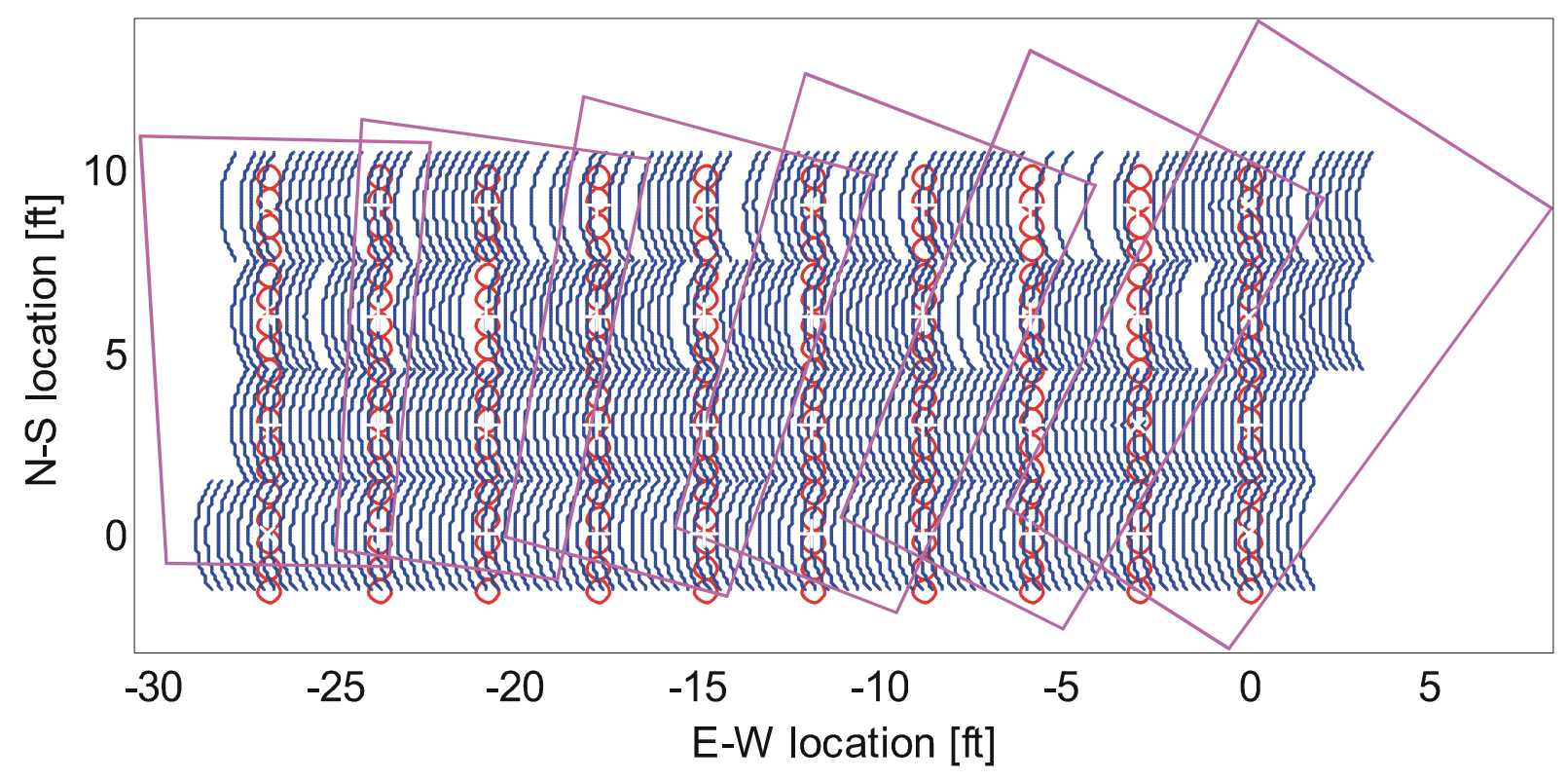

Figure 2. Sample locations for OSU data collection. The vertical columns of circles are EMI sample points (decimated by a factor of four for clarity), the rows of arcs indicate GPR scan locations, and the overlapping quadrilaterals define the boundaries of IR images. Mine-like and clutter-like targets are buried on a uniform grid of size 3 feet in each dimension (see Figure 1).

For the EMI sensor, measurements were performed by scanning South to North over each column of the grid. The metal detector was mounted on a nonmetallic cart with the search head placed about 1.5 inches above the ground. The sensitivity of the metal detector was adjusted to give no tone when the search head was located over a non target region but to produce an audible tone when the test piece (described in Section 2) was about 2 inches from the loop. The cart was placed on a pair of wooden rails. Each scan was started with the search head positioned at 18 inches prior to the start of the grid. The cart was advanced in 2 inch steps and at each location the time waveform and audio tone were recorded. To improve the signal to noise ratio, a digital oscilloscope was used to average 100 waveforms at each position. Data was collected until the search head was 16 inches north of the northern-most edge of the grid.

IR data collection was performed with the camera located atop the two-story ESL building. From this location the mine grid is larger than the field of view of the camera. A sequence of six photos were acquired across the grid to obtain complete coverage and image redundancy. Sequences of photos were acquired at 15 minute intervals throughout the day.

\section{PRE-PROCESSING AND FEATURE EXTRACTION ALGORITHMS}

To prepare the sensor data for fusion, a substantial amount of processing is required. For most sensors, the computational resources required for this processing exceeds that required for fusion.

\subsection{GPR}

As noted previously, stepped-frequency scans are acquired at 48 points located along arcs of 38 inch length. These data, which comprise complex samples at equally spaced frequencies, are first processed to subtract the system background response. The data are then windowed and inverse Fourier transformed to the time domain. A software range gate is applied to isolate the time interval containing the desired signal.

A major problem in most GPR data is the ground reflection, which typically dominates the received signal. We employ an iterative technique to eliminate this response. The onset time and duration of the ground reflection are estimated from low-pass filtered down-range (depth) profiles. The time-domain impulse response of the system is 
estimated and then iteratively subtracted from the data at points within the ground reflection window. The process is effective, but care must be taken that near-surface targets are not also removed in the process.

Features used for the GPR include the cumulative energy (after suppression of the ground reflection) and the late-time spectra both as a function of sample position. The presence of multiple reflections between the mine and the ground surface or between the top and bottom surface of dielectric mines is evident in some data and appears in the late-time spectra.

\subsection{EMI}

The EMI sensor can output both time-domain waveforms directly from the sensor head and an internally processed signal that is provided to the user as an audio tone. The time-domain data is potentially more informative, and we have concentrated our initial work on that output. The usefulness of that data, however, has not met our expectations, and we are reconsidering our approach.

The principal feature derived from the time-domain data is a pointwise estimate of the waveform decay rate from the background-subtracted waveform. Several direct and indirect estimates of this quantity can be formed, including (1) the mean and standard deviation of each trace, (2) an integral of the decay profile with respect to time, (3) the value of the last point in the sample window, and (4) a multiple window method, in which ratios of the mean signal in three temporal windows are computed. After examining the output of each of these methods we found them to be highly correlated, with little difference in classifier performance.

Because of slow naturally occurring variations in the sensor background, it is useful to process these point feature estimates along a sensor track. Such processing helps to remove the background trends and it increases signal detectability. A similar technique has been used ${ }^{1}$ to improve the performance of another EMI sensor. The algorithm was implemented using a high-pass filter with a cutoff frequency selected to enhance detectability of the smallest objects present.

\subsection{IR}

The IR data requires fairly extensive processing. After converting the camera data from the vendor's proprietary data format to temperature values, we perform a perspective remapping to transform the data to ground coordinates. This transformation is required to locate ground positions for subsequent fusion. The images do not cover the entire mine grid, and we currently process them individually rather than form a single full-scene mosaic.

IR images of natural scenes contain both fine-scale emissivity variations and large scale variations in illumination and scene content. To mitigate these phenomena we have employed a wavelet-based algorithm comparable to a spatial bandpass filter. The procedure involves forming the 2-D discrete wavelet transform of the image and dropping coefficients at spatial scales outside of the target band. This type of nonlinear editing of wavelet coefficients for noise suppression or image compression has been extensively developed in the literature ${ }^{2}{ }^{3}$ The use of wavelets for detecting changes in trends is a closely related function and has also received attention. ${ }^{4}$ Recently, both techniques were used in a demining context. ${ }^{5}$

The final processing step is feature extraction. The process begins with the detection of mine-like regions via a suitable filter. The filter is formed from a uniform circular disk with a concentric negative outer ring. The inner ring approximates a matched filter, and the outer ring imposes a penalty for non-circular shapes. From the filter output we identify regions of high and low temperature extremes. Thresholding these data produces a binary (segmented) image, which is the basis for further feature extraction. The resulting binary image contains "blobs" which comprise both true and false targets. Some of these blobs are the result of edge effects in the filtering operations described above. We reduce the number of false alarms by eliminating small blobs and by rejecting blobs on the edges. Within each of the segmented regions we compute the following characteristics, which comprise its feature vector: (1) blob area, (2) blob perimeter pixel count (an approximation to perimeter length), (3) bounding rectangle dimensions, (4) centroid location, (5) mean temperature offset in the original image, and (6) variance (a simple texture estimator).

\section{FUSION ALGORITHM}

Sensor position data are important for sensor fusion, since to combine data one must be reasonably confident that they represent views of the same physical location. As a result, position-related issues have a major role in determining the success of fusion. In particular, we note the following problems: 
First, most countermine sensors produce ambiguous target position estimates in one or more dimensions. EO sensors sample points on the surface but are ambiguous in depth. Other sensors are sampled along tracks, typically along (reasonably) parallel lines at nearly equal intervals. GPR sensors typically sample depth and one along-track dimension. EMI sensors sample only in the along track-dimension.

Second, as noted previously, the locations of sample points are often not coincident. The sensors involved may be deployed on different platforms, or it may be impractical (or simply inconvenient) to sample all sensors at the same spatial location.

Finally, errors in positioning often arise when moving platforms are used. Such errors occur even in carefully conducted field experiments, and fusion algorithms that tolerate these errors are required.

In this section we present a new technique for performing sensor fusion in the presence of these position-related problems. The technique uses probabilistic models of sensor signatures to estimate the probability of various target hypotheses at locations away from the measured data. A modest capability for data extrapolation to points beyond the measurements is also inherent.

\subsection{Fusion of Non-Coincident Samples Without Detection}

Suppose that $N_{S}$ countermine sensors are used to acquire data in a region containing mines, and that sensor $i$ acquires $J_{i}$ data samples $\mathbf{d}_{i j}$ at points $\mathbf{R}_{i j}$. These samples may comprise scalar measurements, vector measurements or image data. In general two sensors $i$ and $i^{\prime}$ will produce samples $\mathbf{d}_{i j}$ and $\mathbf{d}_{i^{\prime} j}$ that are different in number, dimensionality (i.e., scalar versus vector measurements), and sample positions $\mathbf{R}_{i j}$ and $\mathbf{R}_{i^{\prime} j}$. For each sensor $i$ and sample position $\mathbf{R}_{i j}$ we extract feature vectors $\mathbf{X}_{i}\left(\mathbf{R}_{i j}\right), j=1,2, \ldots, J_{i}$. (In some cases the features may be derived from a sequence of data records around each point $\mathbf{R}_{i j}$.) Let the set of all features acquired in the area be given by

$$
\Omega=\left\{\mathbf{X}_{i}\left(\mathbf{R}_{i j}\right), i=1, \ldots, N_{s} ; j=1, \ldots, J_{i}\right\}
$$

Let $\mathbf{R}$ be any point at which we wish to determine the presence of a mine. This point need not be a sample point or even within the sample region. We form $K$ hypotheses $H_{k}(\mathbf{R})$ regarding the presence or absence of various types of mines and clutter at $\mathbf{R}$. As usual, the set of hypotheses $\left\{H_{k}(\mathbf{R}), k=1,2, \ldots, K\right\}$ must be complete, i.e., it must encompass all possible decisions regarding the presence of a mine.

Our goal is to evaluate the a posteriori probabilities $\operatorname{Pr}\left(H_{k}(\mathbf{R}) \mid \Omega\right)$ when the features $\Omega$ were not necessarily acquired at the point $\mathbf{R}$. Without loss of generality we cam define our coordinate system such that $\mathbf{R}=0$. To simplify the notation in what follows we define the composite feature vector at some point $\mathbf{R}^{\prime}$

$$
\begin{aligned}
\mathcal{X}\left(\mathbf{R}^{\prime}\right) & =\left[\begin{array}{c}
\mathbf{X}_{1}\left(\mathbf{R}^{\prime}\right) \\
\mathbf{X}_{2}\left(\mathbf{R}^{\prime}\right) \\
\vdots \\
\mathbf{X}_{N_{s}}\left(\mathbf{R}^{\prime}\right)
\end{array}\right] \\
\mathcal{X}_{0} & =\mathcal{X}(0)
\end{aligned}
$$

We will assume that a classifier has been trained using data acquired over known mines to produce the densities

$$
f_{\mathcal{X}_{0} \mid H_{k}(0)}\left(\mathcal{X}_{0} \mid H_{k}(0)\right)=f_{\mathcal{X}_{0} \mid H_{k}(0)}\left(\mathbf{X}_{1}(0), \mathbf{X}_{2}(0), \ldots, \mathbf{X}_{N_{s}}(0) \mid H_{k}(0)\right)
$$

where we use the notation $f_{\mathcal{X}}\left(\mathcal{X}^{\prime}\right)$ for the probability density function of random variable $\mathcal{X}$ evaluated at $\mathcal{X}^{\prime}$.

We can relate $f_{\mathcal{X}_{0} \mid H_{k}(0)}$ to the available data $\Omega$ as follows: Elementary properties of conditional densities permit us to write

$$
\operatorname{Pr}\left(H_{k}(0) \mid \Omega\right)=\int d \mathcal{X}_{0} \operatorname{Pr}\left(H_{k}(0) \mid \mathcal{X}_{0}, \Omega\right) f_{\mathcal{X}_{0} \mid \Omega}\left(\mathcal{X}_{0} \mid \Omega\right)
$$

For each sensor $i$ there exists a distance $\Delta_{i}$ such that for all mines of interest the sensor response is insignificant outside of this radius. For each point $\mathbf{R}$ we can then define a local collection of feature vectors $\mathcal{Y}$ that are significant in a neighborhood of $\mathbf{R}$. We write

$$
\mathcal{Y}=\left\{\mathbf{X}_{i j_{m}}, i=1,2, \ldots, N_{s} ; m=1,2, \ldots, M\right\}
$$


where the subsequence $j_{m}, m=1,2, \ldots, M$ defines the points $\mathbf{R}_{i j_{m}}$ that lie close to $\mathbf{R}$. Since only these elements of $\Omega$ contribute to our estimate of $\operatorname{Pr}\left(H_{k}(0) \mid \Omega\right)$ we have

$$
\operatorname{Pr}\left(H_{k}(0) \mid \Omega\right)=\operatorname{Pr}\left(H_{k}(0) \mid \mathcal{Y}\right)=\int d \mathcal{X}_{0} \operatorname{Pr}\left(H_{k}(0) \mid \mathcal{Y}, \mathcal{X}_{0}\right) f_{\mathcal{X}_{0} \mid \mathcal{Y}}\left(\mathcal{X}_{0} \mid \mathcal{Y}\right)
$$

Consider next the integrand in equation (7). The data $\mathcal{Y}$ are most consistent with a value of $\mathcal{X}_{0}$ that (hopefully) corresponds to the true feature vector at $\mathbf{R}=0$. For this value the conditional density $f_{\mathcal{X}_{0} \mid \mathcal{Y}}$ has a maximum, which is the maximum a posteriori (MAP) estimate of $\mathcal{X}_{0}$. At such points we have

$$
\operatorname{Pr}\left(H_{k}(0) \mid \mathcal{Y}, \mathcal{X}_{0}\right) f_{\mathcal{X}_{0} \mid \mathcal{Y}}\left(\mathcal{X}_{0} \mid \mathcal{Y}\right) \approx \operatorname{Pr}\left(H_{k}(0) \mid \mathcal{X}_{0}\right) f_{\mathcal{X}_{0} \mid \mathcal{Y}}\left(\mathcal{X}_{0} \mid \mathcal{Y}\right)
$$

When the data strongly support this estimate, $f_{\mathcal{X}_{0} \mid \mathcal{Y}}$ will be peaked and the accuracy of the approximation is improved. This approximation is employed in what follows, and it leads to

$$
\operatorname{Pr}\left(H_{k}(0) \mid \mathcal{Y}\right) \approx \int d \mathcal{X}_{0} \operatorname{Pr}\left(H_{k}(0) \mid \mathcal{X}_{0}\right) f_{\mathcal{X}_{0} \mid \mathcal{Y}}\left(\mathcal{X}_{0} \mid \mathcal{Y}\right)
$$

Thus, we obtain the intuitive result that the probability of a mine (or the absence of a mine) at position $\mathbf{R}$ given data $\mathcal{Y}$ from adjacent locations is a weighted integral of probabilities given all features $\mathcal{X}_{0}$ that could be acquired over the mine. The weighting factor is the probability of obtaining features $\mathcal{X}_{0}$ given features $\mathcal{Y}$ measured nearby.

Equation (9) has several important limiting cases. If for each sensor $i$ and some sample $j$ the distances $\left|\mathbf{R}-\mathbf{R}_{i j}\right|$ are small compared to the mine's signature radius $\Delta_{i}$, then the measurements made at the nearby sample locations will be highly correlated. When the noise levels are small we have $f\left(\mathbf{X}_{i}(0) \mid \mathbf{X}_{i}\left(\mathbf{R}_{i j}\right)\right) \rightarrow \delta\left(\mathbf{X}_{i}(0)-\mathbf{X}_{i}\left(\mathbf{R}_{i j}\right)\right)$ and

$$
\operatorname{Pr}\left(H_{k}(\mathbf{R}) \mid \Omega\right) \approx \operatorname{Pr}\left(H_{k}(\mathbf{R}) \mid \mathbf{X}_{1}\left(\mathbf{R}_{1 j_{1}}\right), \mathbf{X}_{1}\left(\mathbf{R}_{1 j_{2}}\right), \ldots, \mathbf{X}_{N_{s}}\left(\mathbf{R}_{N_{s} j_{M}}\right)\right)
$$

which implies that a "nearest-neighbor" approach to non-coincident sampling is effective under these conditions. If, however, the distances $\left|\mathbf{R}-\mathbf{R}_{i}\right|$ are large compared to the mine's sphere of influence, then the measurements made at the remote location will not be related to those over the mine. If all sensors satisfy this condition, then $f_{\mathcal{X}_{0} \mid \mathcal{Y}}\left(\mathcal{X}_{0} \mid \mathcal{Y}\right)$ tends to $f_{\mathcal{X}_{0}}\left(\mathcal{X}_{0}\right)$ and we have $\operatorname{Pr}\left(H_{k}(\mathbf{R}) \mid \mathcal{Y}\right) \sim \operatorname{Pr}\left(H_{k}(\mathbf{R})\right)$. In this case the sensors add no information, and fusion will be ineffective.

An important component of equation (9) is the conditional probability density $f_{\mathcal{X}_{0} \mid \mathcal{Y}}\left(\mathcal{X}_{0} \mid \mathcal{Y}\right)$, which we can view as describing the consistency of measured data $\mathcal{Y}$ with the data extrapolation $\mathcal{X}_{0}$. Since the expected sensor output is dependent on the hypotheses employed, it is convenient to introduce conditioning on $H_{k}(0)$. It is straightforward to show

$$
f_{\mathcal{X}_{0} \mid \mathcal{Y}}\left(\mathcal{X}_{0} \mid \mathcal{Y}\right)=\frac{\sum_{k^{\prime}=1}^{K} \operatorname{Pr}\left(H_{k^{\prime}}(0)\right) f_{\mathcal{X}_{0} \mid \mathcal{Y}, H_{k^{\prime}}}\left(\mathcal{X}_{0} \mid \mathcal{Y}, H_{k^{\prime}}(0)\right) f_{\mathcal{Y} \mid H_{k^{\prime}}}\left(\mathcal{Y} \mid H_{k^{\prime}}(0)\right)}{f_{\mathcal{Y}}(\mathcal{Y})}
$$

where the density of $\mathcal{Y}$ is

$$
f_{\mathcal{Y}}(\mathcal{Y})=\sum_{k^{\prime}=1}^{K} \operatorname{Pr}\left(H_{k^{\prime}}(0)\right) f_{\mathcal{Y} \mid H_{k^{\prime}}}\left(\mathcal{Y} \mid H_{k^{\prime}}(0)\right)
$$

We can interpret the components of this result as follows: If $\mathcal{X}_{0}$ represents an interpolation of the data $\mathcal{Y}$ to position $\mathbf{R}=0$, then $f_{\mathcal{X}_{0} \mid \mathcal{Y}, H_{k^{\prime}}}\left(\mathcal{X}_{0} \mid \mathcal{Y}, H_{k^{\prime}}(0)\right)$ is related to the "goodness of fit" of our interpolated value to the data $\mathcal{Y}$, and $f_{\mathcal{Y} \mid H_{k^{\prime}}}\left(\mathcal{Y} \mid H_{k^{\prime}}(0)\right)$ is the consistency of our data with hypothesis $H_{k^{\prime}}$.

Using this result the required conditional probability $\operatorname{Pr}\left(H_{k}(0) \mid \mathcal{Y}\right)$ in equation $(9)$ can be written as a quotient of weighted sums

$$
\operatorname{Pr}\left(H_{k}(0) \mid \mathcal{Y}\right) \approx \frac{1}{f_{\mathcal{Y}}(\mathcal{Y})} \sum_{k^{\prime}=1}^{K} \operatorname{Pr}\left(H_{k^{\prime}}(0)\right) f_{\mathcal{Y} \mid H_{k^{\prime}}}\left(\mathcal{Y} \mid H_{k^{\prime}}(0)\right) I_{k k^{\prime}}(\mathcal{Y})
$$

where

$$
I_{k k^{\prime}}(\mathcal{Y}) \approx \int d \mathcal{X}_{0} \operatorname{Pr}\left(H_{k}(0) \mid \mathcal{X}_{0}\right) f_{\mathcal{X}_{0} \mid \mathcal{Y}, H_{k^{\prime}}}\left(\mathcal{X}_{0} \mid \mathcal{Y}, H_{k^{\prime}}(0)\right)
$$


which is an integral over feature space involving a density function and a classifier.* We can express the classifier in terms of the available density function, which leads to

$$
I_{k k^{\prime}}(\mathcal{Y})=\operatorname{Pr}\left(H_{k}(0)\right) \int \frac{d \mathcal{X}_{0}}{f_{\mathcal{X}_{0}}\left(\mathcal{X}_{0}\right)} f_{\mathcal{X}_{0} \mid H_{k}}\left(\mathcal{X}_{0} \mid H_{k}(0)\right) f_{\mathcal{X}_{0} \mid \mathcal{Y}, H_{k^{\prime}}}\left(\mathcal{X}_{0} \mid \mathcal{Y}, H_{k^{\prime}}(0)\right)
$$

where

$$
f_{\mathcal{X}_{0}}\left(\mathcal{X}_{0}\right)=\sum_{k^{\prime}=1}^{K} \operatorname{Pr}\left(H_{k^{\prime}}(0)\right) f_{\mathcal{X}_{0} \mid H_{k^{\prime}}}\left(\mathcal{X}_{0} \mid H_{k^{\prime}}(0)\right)
$$

To evaluate equations (13) and (15) we require the conditional densities $f_{\mathcal{Y} \mid H_{k}}$ and $f_{\mathcal{X}_{0} \mid \mathcal{Y}, H_{k}}$. Directly over the mine we model the random measured features $\mathbf{X}(0)$ for a given hypothesis $H_{k}$ as a random mine signature $\mathbf{S}_{i k}$ contaminated by zero-mean, additive noise $\mathbf{N}_{i}$, viz:

$$
\mathbf{X}_{i}(0) \mid H_{k}(0)=\mathbf{S}_{i k}+\mathbf{N}_{i}
$$

We take $\mathbf{N}_{i}$ to be independent of the measurement position and of $\mathbf{S}_{i k}$. At points away from the mine, we introduce a multiplicative factor $G_{i k}(\mathbf{R})$ as follows:

$$
\mathbf{X}_{i}(\mathbf{R}) \mid H_{k}(0)=\mathbf{S}_{i k} G_{i k}(\mathbf{R})+\mathbf{N}_{i}
$$

where $G_{i k}$ is a monotonically decreasing ${ }^{\dagger}$ scalar function of $R=|\mathbf{R}|$ with $G_{i k}(0)=1$. The form of the functions $G_{i k}$ will depend on the sensor $i$ and the hypothesis $H_{k}$, but they can be determined from modeling or measurements. Define the mine signature over all sensors for hypothesis $k$ as

$$
\mathbf{S}_{k}=\left[\begin{array}{c}
\mathbf{S}_{1 k} \\
\mathbf{S}_{2 k} \\
\vdots \\
\mathbf{S}_{N_{s} k}
\end{array}\right]
$$

Since $\mathbf{N}_{i}$ is assumed to be zero mean, the mean of $\mathbf{S}_{k}$ is equal to the mean of the feature vector $\mathcal{X}_{0} \mid H_{k}(0)$. An approach to estimating the required densities is to first estimate $\mathbf{S}_{k}$ from the data $\mathcal{Y}$, and then to estimate the density of $\mathcal{Y}$ from the density of $\mathbf{S}_{k}$ using the transformation in equation (18) and the independence of $\mathbf{S}_{k}$ and $\mathbf{N}$.

\subsection{The Fusion Process}

The fusion algorithm defined above involves a number of steps, which we summarize here. The preparatory steps are as follows:

1. Define $K$ hypotheses $H_{k}$ regarding the presence or absence of different types of mines at a point. The hypotheses should be chosen to minimize within-class variations in features.

2. Collect sensor data directly over known mine positions for each of these hypotheses.

3. Use these data to develop estimates of $f_{\mathcal{X}_{0} \mid H_{k}(0)}\left(\mathcal{X}_{0} \mid H_{k}(0)\right)$.

4. Develop models $G_{i k}(\mathbf{R})$ for the features acquired by these sensors under each hypothesis.

Use of the algorithm begins with the collection of measurements

1. Acquire data over an unknown area of interest and form the feature set $\Omega$.

2. Select the point of interrogation $\mathbf{R}$ at which to assess the presence of a mine The vector $\mathbf{R}$ might sequentially sample a grid over the entire area of interest at a spacing comparable to that of the smallest expected mine signature.

\footnotetext{
${ }^{*}$ Equation (14) becomes an exact result of equation (5) if we write $\operatorname{Pr}\left(H_{k}(0) \mid \mathcal{X}_{0}\right) \rightarrow \operatorname{Pr}\left(H_{k}(0) \mid \mathcal{Y}, \mathcal{X}_{0}\right)$

${ }^{\dagger}$ It is implicitly assumed here that the features change monotonically when we move away from the mine. Features with non-monotonic behavior (e.g., raw magnetometer signatures) require a more complicated treatment.
} 
3. Identify the points $\mathbf{R}_{i j_{m}}, m=1,2, \ldots, M$, close to $\mathbf{R}$, and form the local feature set $\mathcal{Y}(\mathbf{R}) \subset \Omega$.

4. Estimate $\operatorname{Pr}\left(H_{k}(\mathbf{R}) \mid \mathcal{Y}(\mathbf{R})\right)$ for all $k=1,2, \ldots, K$. In evaluating these expressions, employ the feature models $G_{i k}$ to obtain the density functions away from the mine.

5. Report these probabilities at each point $\mathbf{R}$.

Details of the process are described in the following sections.

\subsection{Feature Models}

Models for the feature distance dependence function $G_{i k}(\mathbf{R})$ can be developed from physical insight or experimental measurements. We assume that the features are linearly related to the data, which makes their amplitude dependence comparable to that of the sensor signature. On the basis of such measurements, we propose the simple model

$$
G_{i k}(\mathbf{R})=\frac{1}{1+\left(|\mathbf{R}| / \Delta_{i k}\right)^{\beta_{i k}}}
$$

where $\Delta_{i k}$ and $\beta_{i k}$ are constants determined by the sensor and the hypothesis. We take $G_{i k}=0$ for the no-mine hypothesis. The quantity $\Delta_{i}$ determines the spatial scale of the signature while $\beta_{i}$ determines its rate of decrease away from the mine. For a metal detector, values for $\Delta_{i}$ are on the order of the loop radius, and $\beta_{i}=6$. For a focussed beam GPR, we find $\Delta_{i}$ comparable to the beam radius and $\beta_{i}=2$.

\subsection{Calculation of Probability Density Functions}

Determining the probability density functions $f_{\mathcal{X}_{0} \mid H_{k}}$ is a crucial part of this work. From these functions and the feature models above we estimate $f_{\mathcal{X}(\mathbf{R}) \mid H_{k}}$ as well. In addition, because $f_{\mathcal{X}_{0} \mid H_{k}}$ is integrated over the features space, it is important that its evaluation be reasonably efficient.

We have used a radial basis function (RBF) neural network to estimate $f_{\mathcal{X}_{0} \mid H_{k}}$. The PDF approximation produced by a RBF NN is of the form

$$
f_{\mathcal{X}}(\mathcal{X})=\sum_{q=1}^{Q} w_{q} f_{q}\left(\left|\mathcal{X}-\mu_{q}\right|\right)
$$

where $\mu_{q}$ are cluster points derived from the data, $Q$ is the number of such points, $w_{q}$ are a set of scalar weighting coefficients, and $f_{q}$ are a family of functions that depend only on the radial distance between the input argument and the cluster point. We have used multi-variate Gaussian functions for the $f_{q}$, where each has a different covariance matrix and the mean of the Gaussian is the cluster centroid.

Training such a RBF comprises finding the cluster points, the weights, and the covariance matrices of the clusters. A variety of algorithms have been suggested for this purpose, and we have employed a learning vector quantization (LVQ) neural network. In our technique the LVQ network is used to determine the classes of the training data. From the class membership we estimate mean and covariance matrices. The weights are determined by the number of points assigned to the cluster. To improve the continuity of the resulting density function, one can slightly expand the covariance matrix for each cluster. ${ }^{6}$ The result is an expression of the form

$$
f_{\mathcal{X} \mid H_{k}}\left(\mathcal{X} \mid H_{k}\right)=\sum_{q=1}^{Q} w_{q} \frac{1}{(2 \pi)^{L / 2} \operatorname{det}\left(\boldsymbol{\Sigma}_{q k}\right)^{1 / 2}} e^{-\left(\mathcal{X}-\mu_{q k}\right)^{T} \cdot \boldsymbol{\Sigma}_{q k}^{-1} \cdot\left(\mathcal{X}-\mu_{q k}\right) / 2}
$$

where $L$ is the length of the feature vector $\mathcal{X}$. It is easy to see that if each cluster point $\mu_{q k}$ is shifted by some amount $\mu_{0}$, then the mean of $f_{\mathcal{X} \mid H_{k}}$ shifts by the same amount. 


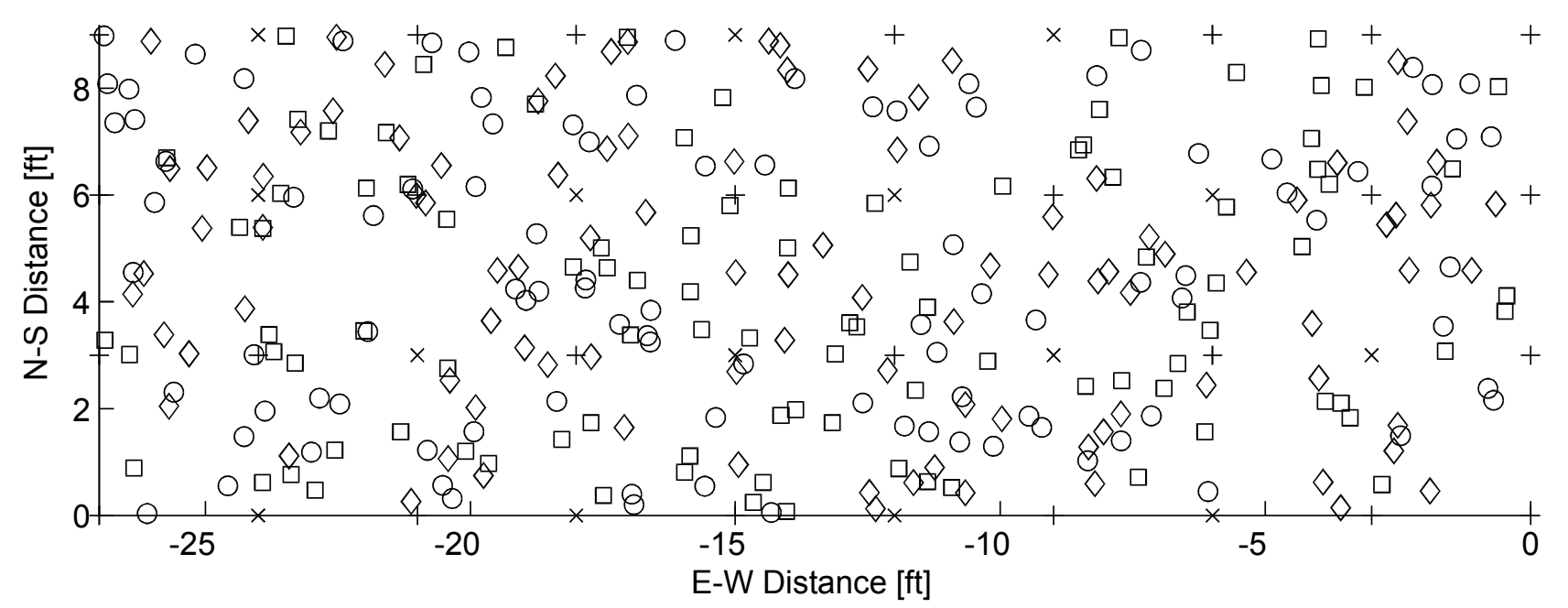

Figure 3. An example of randomly located measurements. The locations of 100 sensor measurements are indicated by the square, circle and diamond markers. Mine and non-mine grid points are indicated by the " $\mathrm{x}$ " and "+" symbols respectively.

\section{RESULTS}

We have developed software to implement the above-described algorithms. Tests of the algorithms were performed using simulated data. Using simulated data in development effectively decouples algorithm problems from problems with ineffective sensors and uninformative features.

To demonstrate the algorithm's insensitivity to measurement locations, we will use randomly placed sensor measurements, an example of which is shown in Figure 3. In that figure 100 measurements are shown for each of three sensors. The sensors are defined to be sensitive out to distances $\Delta_{i}=12$ inches. Two classes, mine and no-mine, are considered. One feature is defined for each of the three sensors, and zero-mean Gaussian noise is added to the simulated features. As a test of the system's ability to deal with uninformative sensors, one of the three features is constrained to provide no useful information.

We interrogate the data set at points $\mathbf{R}$ located on a 9 inch grid. At each point, the nearest $M=3$ adjacent measurements for each sensor were identified and used to form $\mathcal{Y}$. The probabilities $\operatorname{Pr}\left(H_{k}(\mathbf{R}) \mid \mathcal{Y}\right)$ were computed using equation (13) and the log-likelihood ratio was formed. The results are shown in Figure 4 for the case when the distance between features (in feature space) is 4 times the noise standard deviation. In the interest of brevity we will refer to this as a "feature-to-noise" ratio of 4 . All the mines are clearly detected in this result. Because the algorithm outputs the probabilities $\operatorname{Pr}\left(H_{k} \mid \Omega\right)$ at each desired point $\mathbf{R}$, we are able to determine the confidence that a mine does or does not exist at every point. This is in contrast with a conventional association-based feature-fusion, which would only provide that information at discrete points where the sensors have made a detection.

Receiver operating characteristic (ROC) curves for several sample densities are shown in Figure 5. Feature-tonoise ratios of 4 and 2 are used in parts (A) and (B) respectively. Performance improves with increasing sample density as shown in the figures. The case of 100 samples comprises an average sample density of $2.4 \mathrm{ft}^{2} /$ sample, which is only marginally better than the sensor's region of sensitivity $\left(3.1 \mathrm{ft}^{2}\right)$. As expected, performance also improves with decreased noise. Essentially perfect performance is achieved at high sample densities and high feature-to-noise ratios.

The algorithm has also been tested using experimentally measured data. The data collection depicted in Figure 2 was processed to extract samples near each of the grid positions. The results are shown in Figure 6 . These data were partitioned into training and testing sets, and the fusion algorithm described above was applied. Because of poor performance by the IR sensor, ${ }^{\ddagger}$ the IR data was excluded from this tests. Because of the limitations of the resulting

\footnotetext{
$\ddagger$ In our first round of data collection the mine field was covered with grass which tends to obscure thermal signatures of buried targets. This grass cover has now been removed.
} 


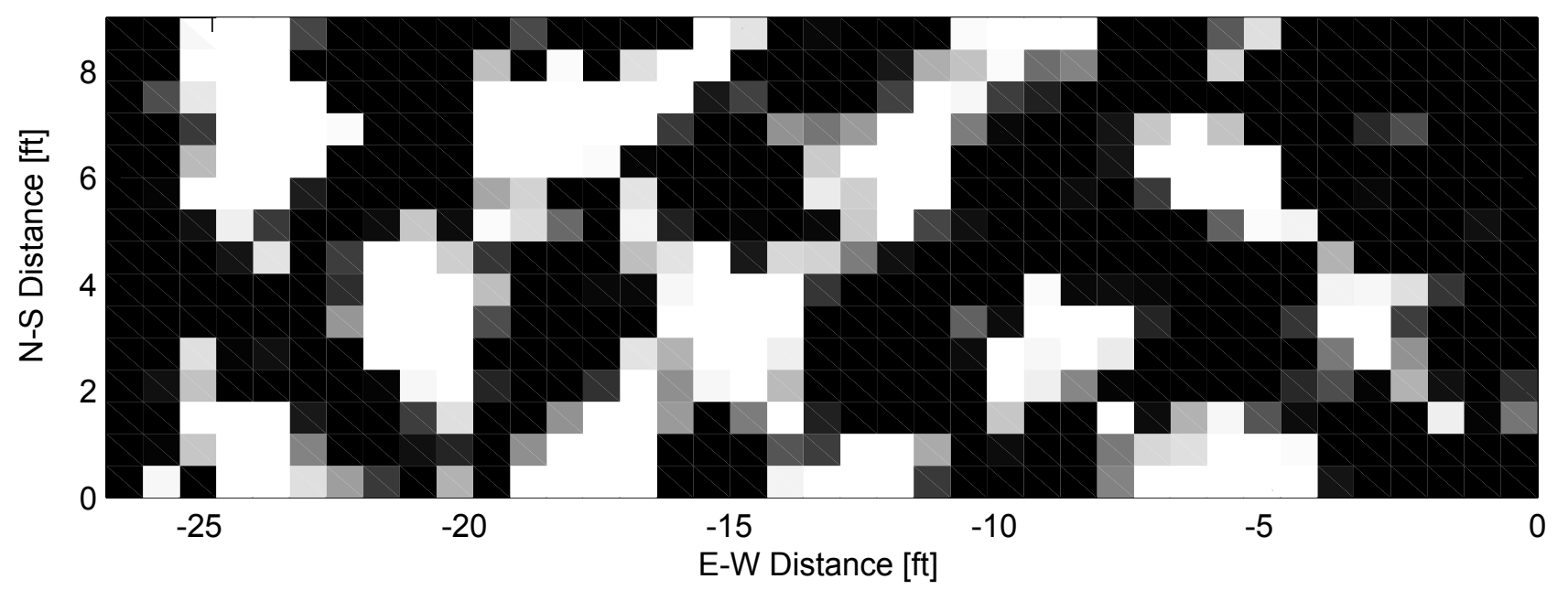

Figure 4. Log-likelihood ratio for fusion of 400 randomly located sensor measurements.

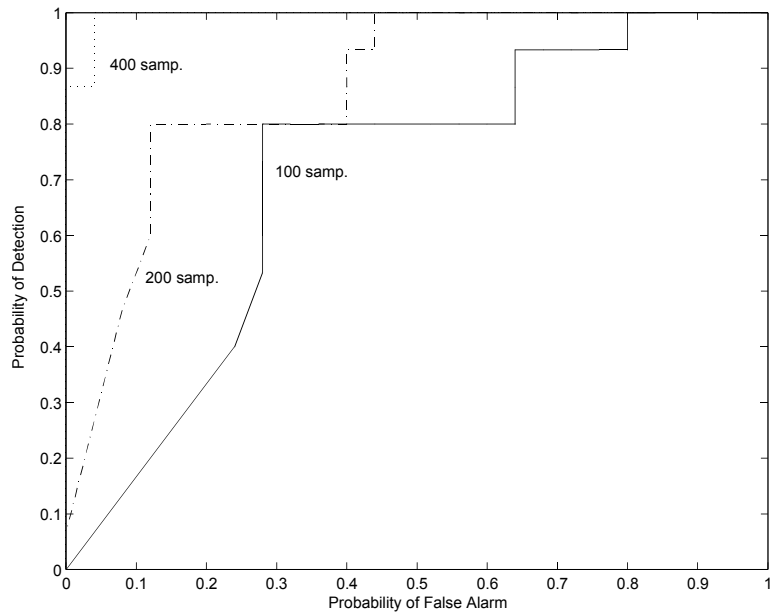

(A)

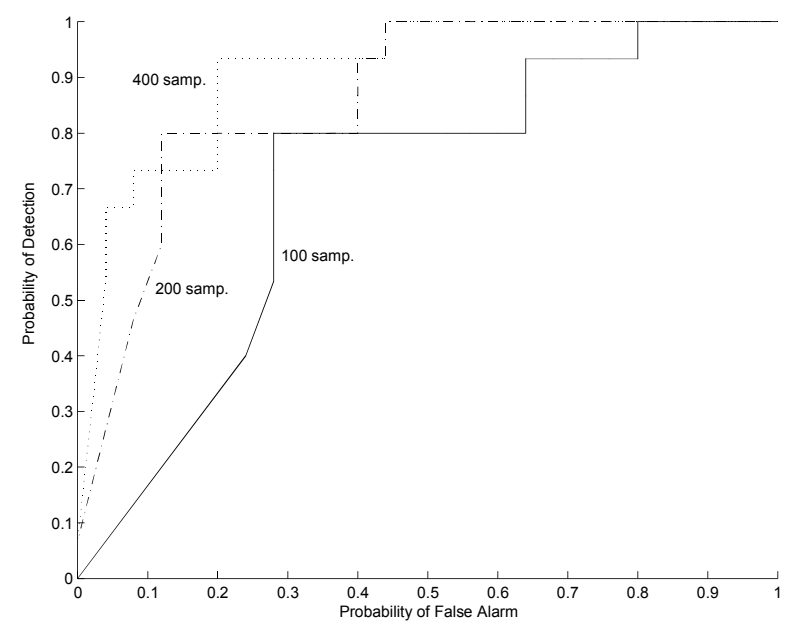

(B)

Figure 5. ROC curves for fusion of randomly located sensor measurements with different densities. The ratio of inter-class distance (in feature space) and noise standard deviation is 4:1 and 2:1 for Figures (A) and (B) respectively. 


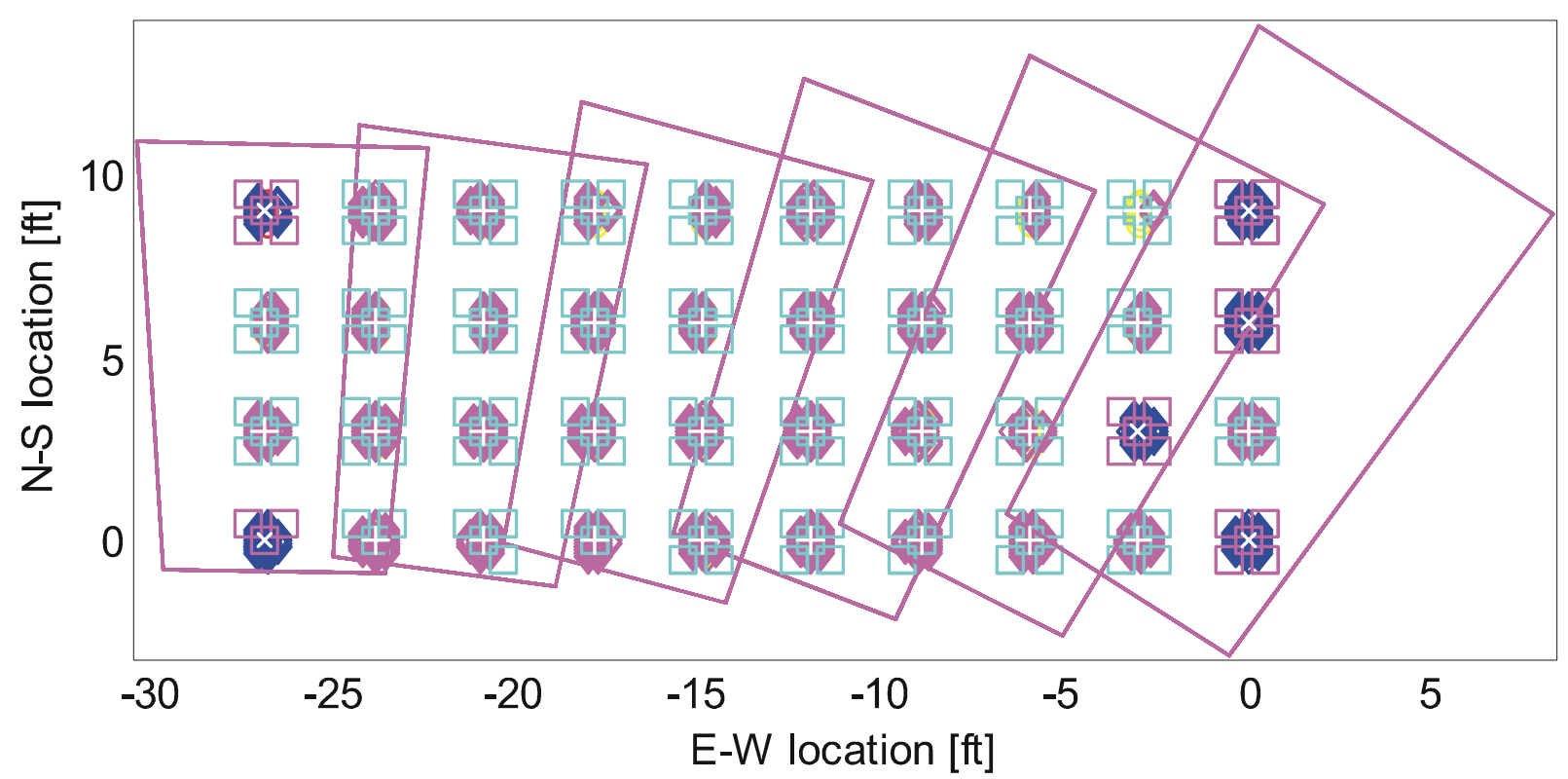

Figure 6. Training and testing data set. The dark masses on the grid points indicate the locations of relevant GPR and EMI data. The small square patches represent IR image chips. Because of overlapping IR imagery, multiple chips exist for some locations.

sensor suite, a two-class problem was defined, comprising metallic mines and non-metallic objects.

The performance of the fused EMI-GPR sensor suite is excellent. The ROC curve for this suite is shown in Figure 7. The benefits of fusion are evident from Figures 8 and 9, where we display the results of EMI-only and GPR-only detection. These results suggest that fusion can be highly effective, but because our data set is relatively small additional testing must be performed to verify this conclusion.

\section{CONCLUDING REMARKS}

We have developed a novel algorithm for fusion of sensor data. The algorithm is insensitive to non-coincident sampling and it does not require individual sensors to perform detection prior to fusion. To support the development of a fusion system based on this and other fusion techniques, pre-processing algorithms have been developed and tested. Measured data have been collected to support testing. Future efforts will focus on improvements and refinements of the algorithms.

\section{ACKNOWLEDGMENTS}

This work was funded by the US Army Research Office under the Multi-University Research Initiative (MURI) on Humanitarian Demining, contracts DAAH04-96-1-0448 and 97-SC-ARO-1015.

\section{REFERENCES}

1. L. Collins, V. George, T. Altshuler, L. Nolte, and L. Carin, "An improved decision-theoretic approach to the optimum detection of mines," in Detection and Remediation Technologies for Mines and Minelike Targets II, A. C. Dubey and R. L. Barnard, eds., SPIE 3079, pp. 716-723.

2. R. R. Coifman and M. V. Wickerhauser, "Wavelets and adapted waveform analysis," in Wavelets: Mathematics and Applications, J. Benedetto and M. Frazier, eds., pp. 399-424, CRC Press, Boca Raton, FL, 1993.

3. D. L. Dohono, I. Johnstone, G. Kerkyacharian, and D. Picard, "Wavelet shrinkage: Asymptopia?," J. Royal Stat. Soc., series B 57(2), pp. 301-369, 1995. 


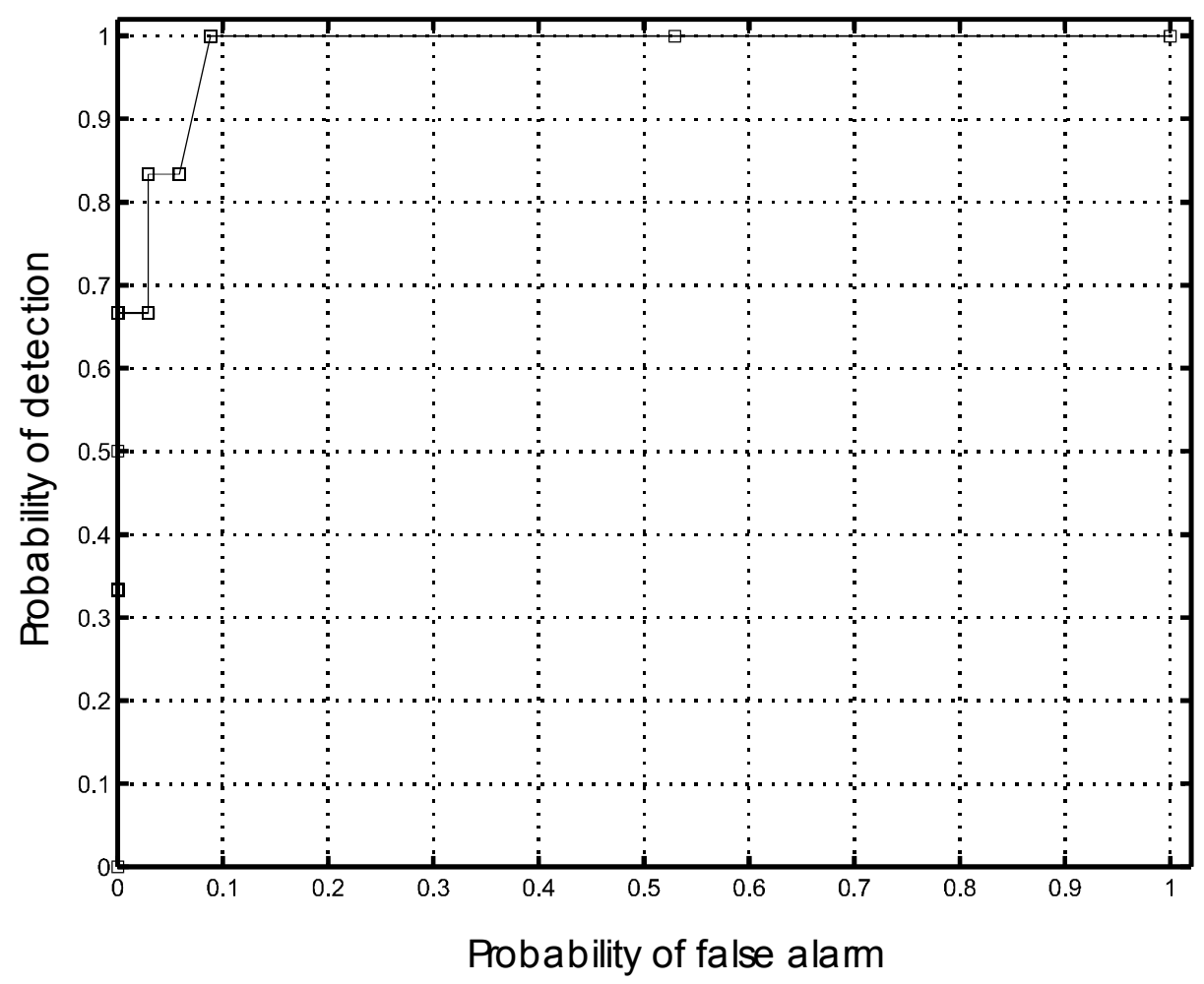

Figure 7. ROC curve for fused EMI-GPR sensor suite.

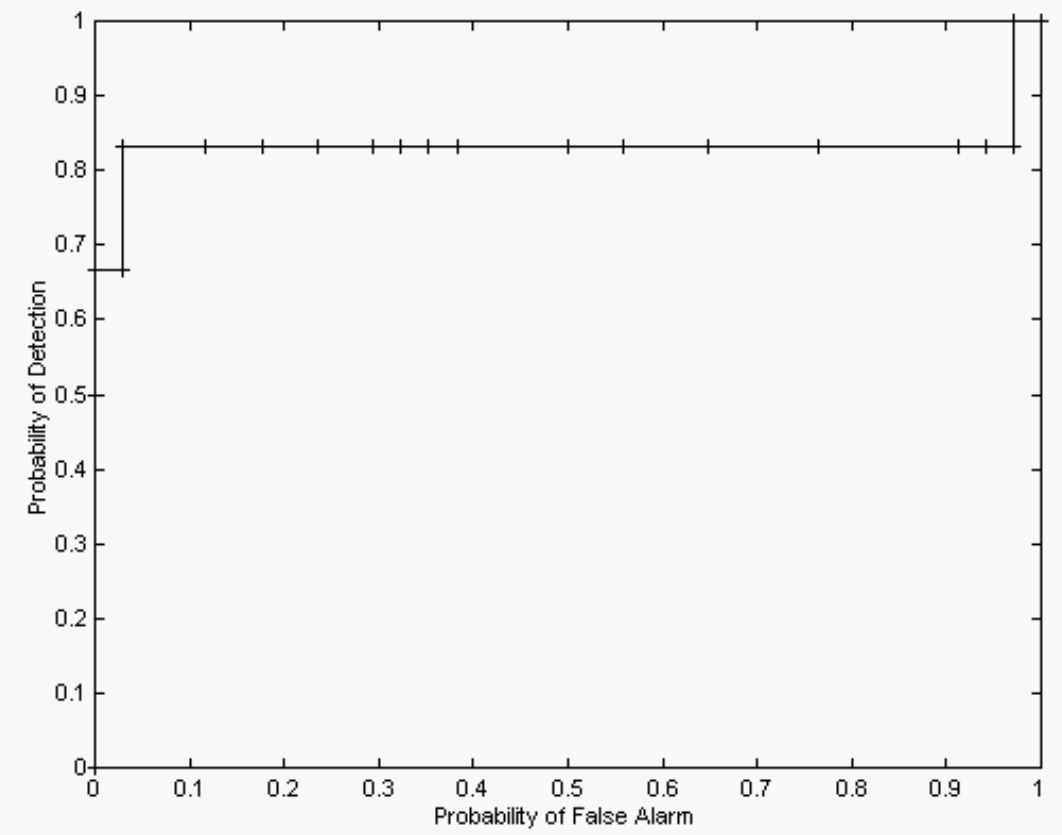

Figure 8. ROC curve for the EMI sensor only. 


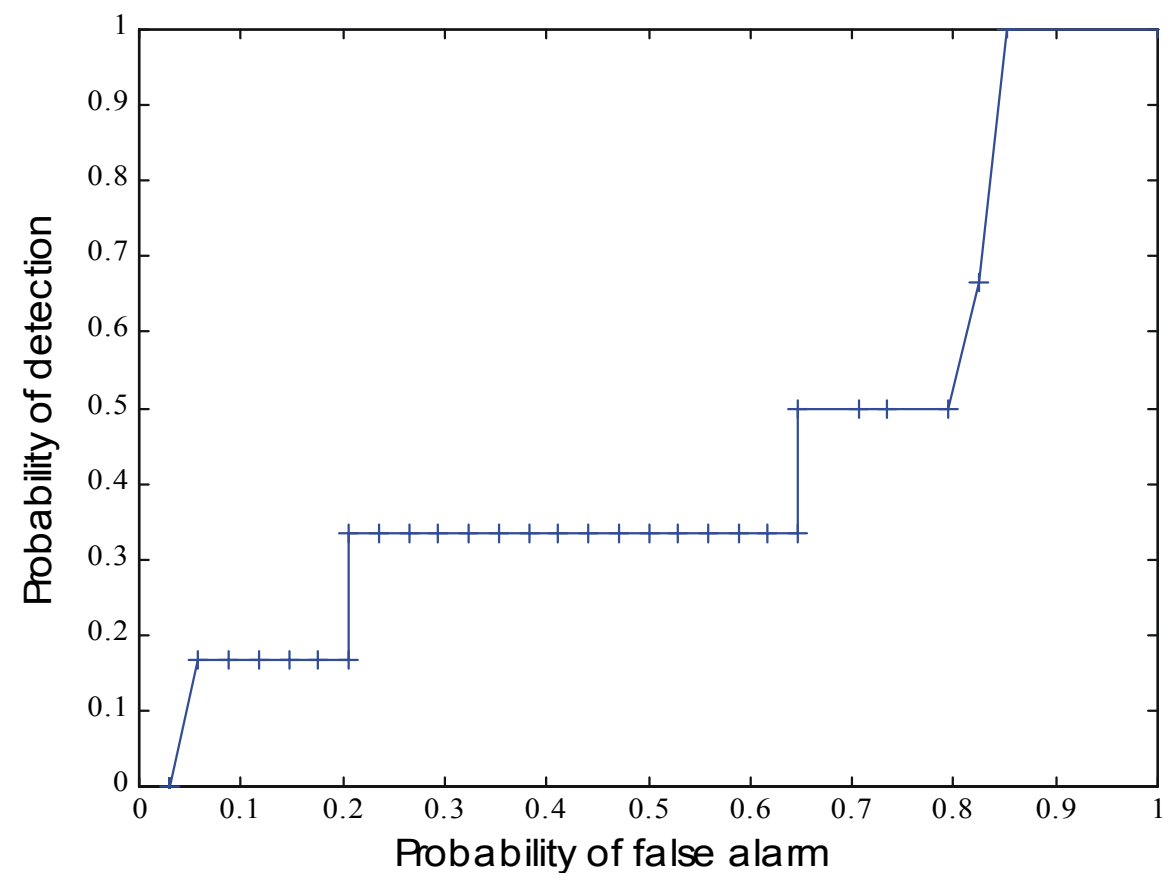

Figure 9. ROC curve for the GPR sensor alone.

4. U. Wiklund, M. Akay, and U. Niklasson, "Short-term analysis of heart-rate variability by adapted wavelet transforms," IEEE Engineering in Medicine and Biology Mag. 16(5), pp. 113-118, 1997.

5. T.-H. Chao, B. Lau, A. Yacoubian, and G. Henderson, "Mine detection using wavelet processing of electro-optic active sensor data," in Detection Technologies for Mines and Minelike Targets, A. Dubey, I. Cindrich, J. Ralston, and K. Rigano, eds., SPIE 2496, pp. 17-21.

6. J. Schurmann, Pattern Classification, John Wiley, New York, 1996. 